\title{
Molecular characterization of pulmonary sarcomatoid carcinoma: analysis of 33 cases
}

\author{
Simone BSP Terra, Jin S Jang, Lintao Bi, Benjamin R Kipp, Jin Jen, Eunhee S Yi and \\ Jennifer M Boland
}

Division of Anatomic Pathology, Department of Laboratory Medicine and Pathology, Mayo Clinic, Rochester, MN, USA

\begin{abstract}
Several targetable genetic alterations have been found in lung cancer, predominantly in adenocarcinomas, which have led to important therapeutic advancements with the advent of targeted therapy. In contrast, the molecular features and presence of targetable genetic abnormalities in pulmonary sarcomatoid carcinomas are largely unknown. Thirty-three cases of pulmonary sarcomatoid carcinoma were tested for approximately 2800 mutations in 50 oncogenes and tumor-suppressor genes, including EGFR, KRAS, NRAS, TP53, BRAF, ERBB2, JAK3, AKT1, ATM, MET, KIT, and PIK3CA. ALK immunostaining was performed, and ALK FISH was performed on cases with any degree of staining. Twenty-four of the 33 cases (72\%) had at least one genetic abnormality: 19 cases (58\%) had TP53 mutations; 10 cases (30\%) had KRAS mutations; AKT1, JAK3, BRAF, NRAS, and PIK3CA mutations were observed in 1 case each (3\%). Six of the 19 cases $(32 \%)$ with a mutation in TP53 had simultaneous mutations in KRAS (18\%). The cases with alterations in JAK3, BRAF, and NRAS also had mutations in TP53. The case showing a mutation in PIK3CA had a mutation in KRAS. No EGFR mutations were observed. One case had $A L K$ gene rearrangement. $A L K$ rearrangement was observed in a single case of sarcomatoid carcinoma ( $3 \%)$, which has currently available targeted therapy. Four tumors had mutations in genes with experimental molecularbased therapy, including BRAF, NRAS, PIK3CA, and AKT1. Testing for targetable mutations should be considered for patients with pulmonary sarcomatoid carcinoma, as a subset may benefit from currently approved drugs or clinical trials of novel therapeutic options available for other types of lung cancer.

Modern Pathology (2016) 29, 824-831; doi:10.1038/modpathol.2016.89; published online 13 May 2016
\end{abstract}

Pulmonary sarcomatoid carcinoma is a rare subtype of lung carcinoma, which is aggressive and poorly differentiated. ${ }^{1-7}$ The poorly differentiated nature is reflected by the presence of at least $10 \%$ tumor giant cells or spindle-shaped cells, hence the designation as 'sarcomatoid'. The sarcomatoid areas may be observed as a form of progression of other types of lung carcinoma, or the entire tumor may be composed of sarcomatoid morphology. Areas of differentiated sarcoma may be observed, mostly commonly resembling rhabdomyosarcoma, chondrosarcoma, or osteosarcoma, and some examples show primitive/ immature mesenchymal stroma. ${ }^{1,3,8}$ In the current 2015 World Health Organization classification, sarcomatoid carcinomas are divided into five histological subtypes depending on the observed morphology: pleomorphic carcinoma, spindle cell carcinoma, giant cell carcinoma, carcinosarcoma, and

Correspondence: Dr JM Boland, MD, Division of Anatomic Pathology, Department of Laboratory Medicine and Pathology, Mayo Clinic, 200 First Street SW, Rochester, MN 55905, USA. E-mail: boland.jennifer@mayo.edu

Received 30 November 2015; revised 8 April 2016; accepted 14 April 2016; published online 13 May 2016 pulmonary blastoma. ${ }^{8}$ These histological subtypes are useful to pathologists in the recognition of the morphological spectrum of sarcomatoid carcinoma, but they do not appear to have clinical or therapeutic significance. Similar to other types of lung carcinoma, sarcomatoid carcinoma tends to affect older patients with a history of tobacco smoking, and a male predominance has been observed.2,3,5,6,9,10 Treatment is the same as for other lung carcinoma with non-small cell morphology, but sarcomatoid carcinoma tends to have a poor prognosis even in early-stage disease. ${ }^{8}$

Substantial advancements in the treatment of lung cancer have occurred during the past decade, predominantly in adenocarcinomas. This accomplishment is largely due to the discovery of several targetable genetic alterations, leading to more effective therapy as well as a better understanding of cellular and molecular mechanisms of lung adenocarcinoma pathogenesis. Some of these molecular abnormalities have proven to be successful biomarkers and/or drug targets. ${ }^{11-14}$ Currently, tumors with $A L K$ rearrangement and EGFR mutations have molecular targeted therapies approved by the US 
Food and Drug Administration. Crizotinib and ceritinib are approved for use in $A L K$ rearranged tumors, ${ }^{15-17}$ while erlotinib, gefitinib, and afatinib are approved for tumors with EGFR mutations. ${ }^{18-21}$ In the US population, these therapies are applicable to a minority of patients with lung carcinoma, as $A L K$ rearrangement is present in $<5 \%$ of lung adenocarcinomas and EGFR mutations are present in 15-25\% of lung adenocarcinomas. KRAS mutations are present in a substantial number of lung adenocarcinomas in the US population (roughly $25-30 \%$ ), but there are currently no approved targeted therapies for these mutations. Many additional rare mutations, predominantly within tyrosine kinase signaling complexes, have been identified in lung adenocarcioma, including BRAF, NRAS, PIK3CA, AKT1, HER2, MET, KIF5B-RET, PTEN, ROS1, and MEK1. This has resulted in several ongoing clinical trials, which are testing the newest potential therapies targeted at many of these genes..$^{11-14}$

Although the molecular characteristics of the more common subtypes of lung carcinoma have been extensively studied, the molecular features and presence of targetable genetic abnormalities in pulmonary sarcomatoid carcinoma are largely unknown. The currently available chemotherapy for sarcomatoid carcinoma is highly ineffective, ${ }^{4-7}$ and thus targeted therapy is a very attractive therapeutic option to improve outcome. A recent study reported frequent MET exon 14 skipping mutations in pulmonary sarcomatoid carcinomas, which may be targetable, in addition to mutations in other genes without currently available targeted therapy, including TP53 and KRAS. ${ }^{22,23}$ The aim of this study is to survey for potentially targetable genetic abnormalities in 33 cases of pulmonary sarcomatoid carcinoma, with the diagnosis previously confirmed based on morphology and immunohistochemistry. ${ }^{24}$

\section{Materials and methods}

The study was approved by the Mayo Clinic Institutional Review Board. Mayo Clinic surgical pathology archives were searched for cases of pulmonary sarcomatoid carcinoma from 1994 to 2011. All cases were re-reviewed by two pathologists (JMB and SBT), with histological and immunohistochemical confirmation of the diagnosis as previously described. ${ }^{24}$ Cases with ambiguous morphology or immunophenotype were reviewed by a third pathologist (ESY), with consensus diagnosis. Cases felt to represent an alternative diagnosis were excluded, as were cases with insufficient remaining tissue for molecular testing, yielding a final study group of 34 cases.

DNA was extracted from formalin-fixed paraffinembedded tumor samples and applied to the Ion AmpliSeq Cancer Hotspot Panel v2 to test by nextgeneration sequencing for approximately 2800 individual mutations in 50 oncogenes and tumor- suppressor genes, including EGFR, KRAS, NRAS, TP53, BRAF, ERBB2, JAK3, AKT1, ATM, MET, KIT, and PIK3CA, as previously described.$^{25}$ Mutational analysis was attempted in 34 cases, but 1 case was excluded owing to technical failure.

ALK immunohistochemistry was performed on formalin-fixed paraffin-embedded tumor sections from 34 cases (clone D5F3, rabbit monoclonal anti-human ALK, Cell Signaling Technology, Danvers, MA, USA). Fluorescent in situ hybridization using a break-apart probe for $A L K$ rearrangement was performed on cases with any degree of ALK immunoreactivity.

Fluorescent in situ hybridization testing was also performed using a break-apart probe for ROS1 rearrangement. This was performed on cases that did not have KRAS mutations or ALK rearrangements, as these genetic abnormalities are generally mutually exclusive of ROS1 rearrangement. One case did not have sufficient tissue remaining for this analysis, so results were obtained on 21 cases.

\section{Results}

Complete patient characteristics, clinical information, and immunohistochemical profile of the majority of the study cases have been previously reported. ${ }^{24}$ In brief, 34 patients were 20 men and 14 women, with mean age of 69 years (range, 46-93). Most patients $(32,94 \%$ ) had a history of cigarette smoking (average 36 pack years; range, 5-150). Two were never smokers. Per current World Health Organization criteria, cases were composed of 23 pleomorphic carcinomas, 8 spindle cell carcinomas, 2 carcinosarcomas, and 1 giant cell carcinoma. Follow-up ranged from 1 to 112 months (mean 25.5 months). At last follow-up, 25 of the 34 patients were deceased $(73.5 \%)$. Twenty patients $(61 \%)$ had died of lung cancer, and the cause of death in the other 5 patients could not be confirmed. Nine patients were alive at last follow-up, 2 of which had persistent disease, and the remaining 7 (20.6\%) were alive without evidence of disease.

A summary of mutational analysis results are presented in Tables 1 and 2 and Figures 1 and 2 . No obvious genetic differences were noted between the different histological subtypes of sarcomatoid carcinoma. Twenty-four of the 33 cases (72\%) showed at least one detectable genetic abnormality, and 9 cases $(28 \%)$ had none of the tested mutations. Of the patients with no detectable mutations, 1 was a never smoker and 8 were former or current smokers. The most commonly observed mutations were in TP53 exons 5-8, which were present in 19 patients $(58 \%)$, all of which had a history of cigarette smoking. The next most commonly observed abnormalities were in $K R A S$, which was mutated in 10 cases $(30 \%$, Figure 3). Nine of the 10 KRAS mutations occurred at codon 12 (four G12V; two G12C; two G12D; and one G12A). The remaining KRAS mutation involved Q61H. Eight of the nine 
Table 1 Summary of molecular alterations identified in 33 cases of pulmonary sarcomatoid carcinoma

\begin{tabular}{|c|c|c|c|c|c|c|c|c|}
\hline \multirow{2}{*}{ No. of cases (\%) } & \multicolumn{3}{|c|}{ No available targeted therapy } & \multicolumn{5}{|c|}{ Experimental or FDA-approved molecular-based therapy } \\
\hline & TP53 & KRAS & $J A K 3^{\mathrm{a}}$ & $B R A F$ & NRAS & PIK3CA & $A K T 1$ & $A L K$ \\
\hline $10(30 \%)$ & $\mathrm{x}$ & & & & & & & \\
\hline $3(9 \%)$ & & $\mathrm{x}$ & & & & & & \\
\hline $6(18 \%)$ & $\mathrm{x}$ & $\mathrm{x}$ & & & & & & \\
\hline $1(3 \%)$ & $\mathrm{x}$ & & $\mathrm{x}$ & & & & & \\
\hline $1(3 \%)$ & $\mathrm{x}$ & & & $\mathrm{x}$ & & & & \\
\hline $1(3 \%)$ & $\mathrm{x}$ & & & & $\mathrm{x}$ & & & \\
\hline $1(3 \%)$ & & $\mathrm{x}$ & & & & $\mathrm{x}$ & & \\
\hline $1(3 \%)$ & & & & & & & $\mathrm{x}$ & \\
\hline $1(3 \%)$ & & & & & & & & $\mathrm{x}$ \\
\hline $8(25 \%)$ & & & & & & & & \\
\hline
\end{tabular}

${ }^{\mathrm{a}}$ Variant of unknown significance.

Table 2 Summary of specific mutations observed

\begin{tabular}{|c|c|c|c|c|c|c|c|}
\hline \multicolumn{3}{|c|}{ No available targeted therapy } & \multicolumn{4}{|c|}{ Experimental or FDA-approved molecular-based therapy } & \multirow{2}{*}{ Total } \\
\hline TP53 & KRAS & $J A K 3$ & $B R A F$ & NRAS & PIКЗСА & $A K T 1$ & \\
\hline \multicolumn{8}{|l|}{ C176Y } \\
\hline \multicolumn{8}{|l|}{ L348F, E349 } \\
\hline \multicolumn{8}{|l|}{ G245D } \\
\hline \multicolumn{8}{|l|}{ R213L } \\
\hline \multicolumn{8}{|l|}{ P142H, C141W } \\
\hline \multicolumn{8}{|l|}{ E285K } \\
\hline \multicolumn{8}{|l|}{ Q104 } \\
\hline \multicolumn{8}{|l|}{ G279E } \\
\hline \multicolumn{8}{|l|}{ L194F } \\
\hline \multirow[t]{4}{*}{ V157F } & & & & & & & $10 / 33(30 \%)$ \\
\hline & G12C & & & & & & \\
\hline & G12D & & & & & & \\
\hline & $\mathrm{G} 12 \mathrm{~V}$ & & & & & & 3/33 (9\%) \\
\hline V157F & G12A & & & & & & \\
\hline R196 & G12C & & & & & & \\
\hline R337L & Q61H & & & & & & \\
\hline R273L & G12D & & & & & & \\
\hline $\mathrm{C} 242 \mathrm{~F}$ & $\mathrm{G} 12 \mathrm{~V}$ & & & & & & \\
\hline R175H & G12V & & & & & & $6 / 33(18 \%)$ \\
\hline $\mathrm{I} 162 \mathrm{~F}$ & & V722I & & & & & $1 / 33(3 \%)$ \\
\hline G105C, R175L & & & D594N & & & & $1 / 33(3 \%)$ \\
\hline \multirow[t]{3}{*}{ T155P, R156P } & & & & A146T & & & $1 / 33(3 \%)$ \\
\hline & $\mathrm{G} 12 \mathrm{~V}$ & & & & E542K & & $1 / 33(3 \%)$ \\
\hline & & & & & & E17K & 1/33 (3\%) \\
\hline
\end{tabular}

patients with KRAS mutations were smokers, while one never smoker had a KRAS G12D mutation. AKT1, BRAF, NRAS, and PIK3CA mutations were observed in one case each (3\%). Of note, the $B R A F$ mutation detected was a non-V600E mutation (D594N). One case had a $J A K 3$ V722I substitution, which is a variant of unknown significance and likely represents a polymorphism. No EGFR mutations were observed.

Ten of the 33 cases (30\%) showed more than one mutation in the tested genes. Six of the 19 cases $(32 \%)$ with a mutation in TP53 had simultaneous mutations in $K R A S(18 \%$, Figure 4). The cases with mutations in $B R A F$ and NRAS also had mutations in TP53, and the case with the $J A K 3$ variant of unknown significance had a mutation in TP53. The case showing a mutation in PIK3CA also had a mutation in KRAS.

One of the 34 cases ( $3 \%$ ) was strongly positive for ALK immunohistochemistry, while all the other cases were completely negative. $A L K$ gene rearrangement was subsequently confirmed by fluorescent in situ hybridization in the immunoreactive tumor (Figure 5), with $A L K$ rearrangement present in 54\% of tumor nuclei. This case showed no additional detected mutations and occurred in a 58-year-old Chinese woman with an 8 pack-year smoking history. She is currently crizotinib naive, as her resected tumor was stage IB and thus adjuvant therapy was not 
initiated. Her tumor showed approximately $40 \%$ spindle cell morphology, while the remaining tumor cells showed solid epithelioid growth pattern with focal signet ring cells. ROS1 fluorescent in situ hybridization was successful in 21 of the 22 cases that lacked $A L K$ rearrangement and KRAS mutations, and all were negative for ROS1 rearrangement.

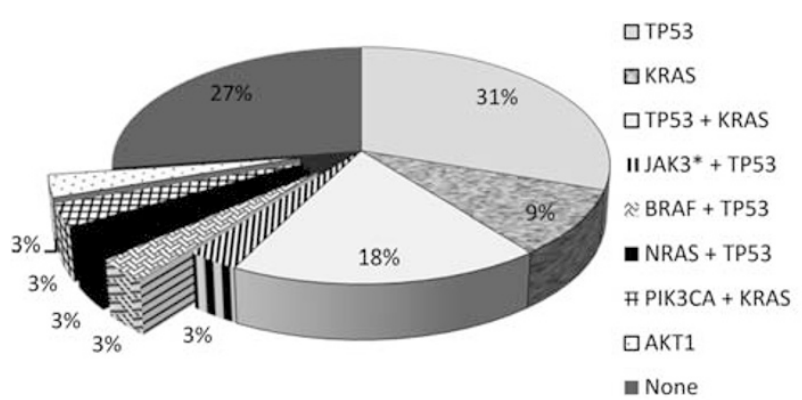

-Variant of unknown significance

Figure 1 Summary of the mutations detected in 33 cases of pulmonary sarcomatoid carcinoma.

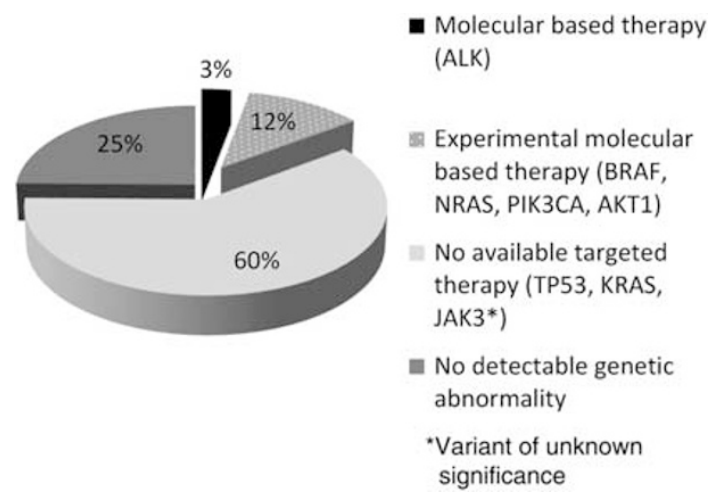

Figure 2 Summary of the targeted therapeutic options for the mutations detected in pulmonary sarcomatoid carcinoma.

\section{Discussion}

Lung cancer has been the most common cause of cancer-related deaths in the worldwide for decades ${ }^{26}$ and still has a very poor prognosis in most cases, as many patients present with advanced stage disease. The advent of targeted therapy has revolutionized tumor testing and treatment strategies for lung adenocarcinoma, based on intense research into the molecular abnormalities driving this disease. However, translating the recent success observed in the treatment of some patients with lung adenocarcinomas to other histological subtypes of lung cancer has been a struggle, as other common types (squamous cell carcinoma and small cell carcinoma) have different molecular alterations. ${ }^{11-14}$ The mutational profile of pulmonary sarcomatoid carcinoma is difficult to establish, given its relative rarity compared with these other more common histological types. However,

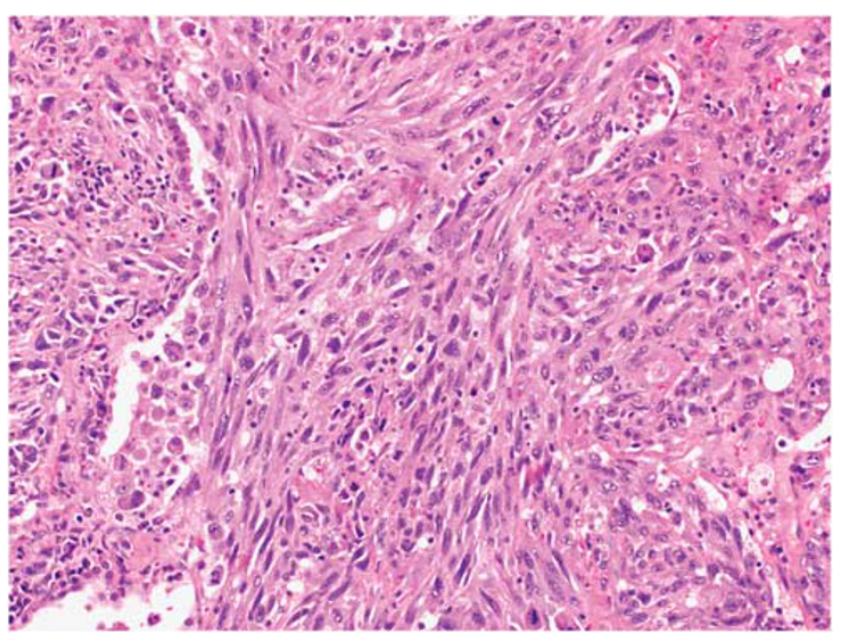

Figure 4 An example of sarcomatoid carcinoma showing both KRAS and TP53 mutations. The tumor consisted exclusively of malignant spindled and pleomorphic cells.
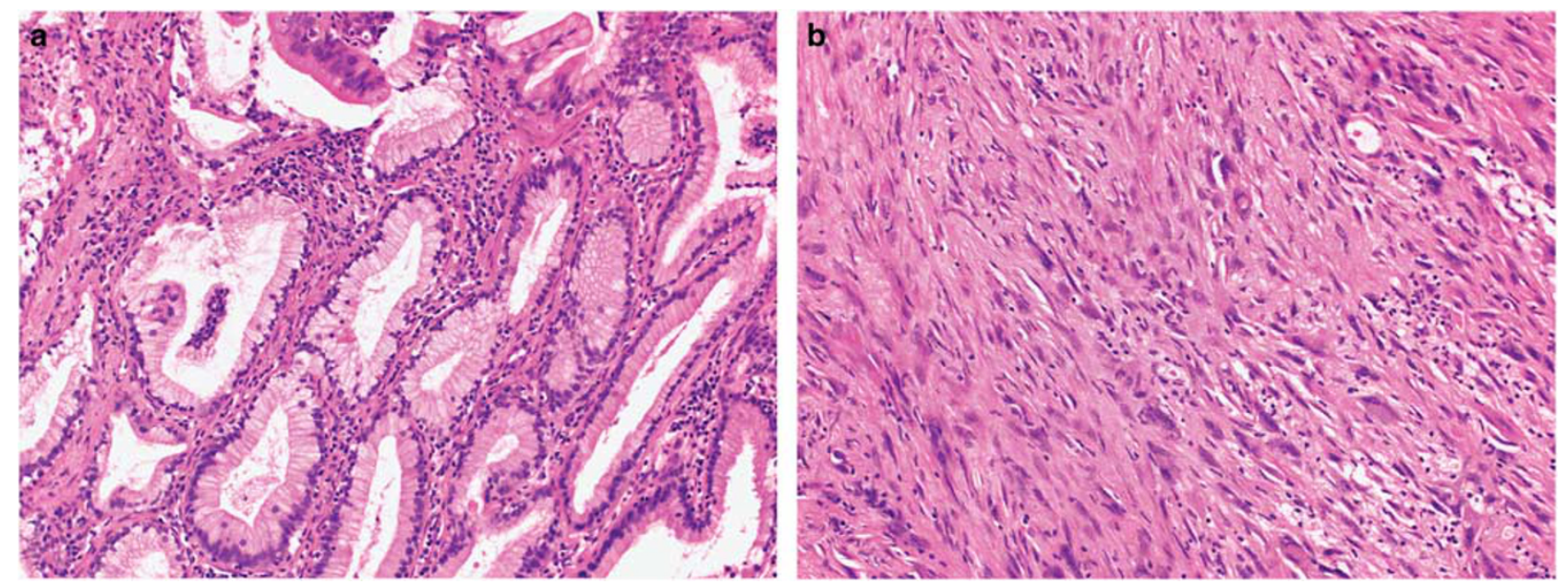

Figure 3 An example of sarcomatoid carcinoma showing KRAS mutation in isolation. The tumor had areas of mucinous adenocarcinoma (a) that transitioned to high-grade spindle cell and giant cell sarcomatoid regions (b). 

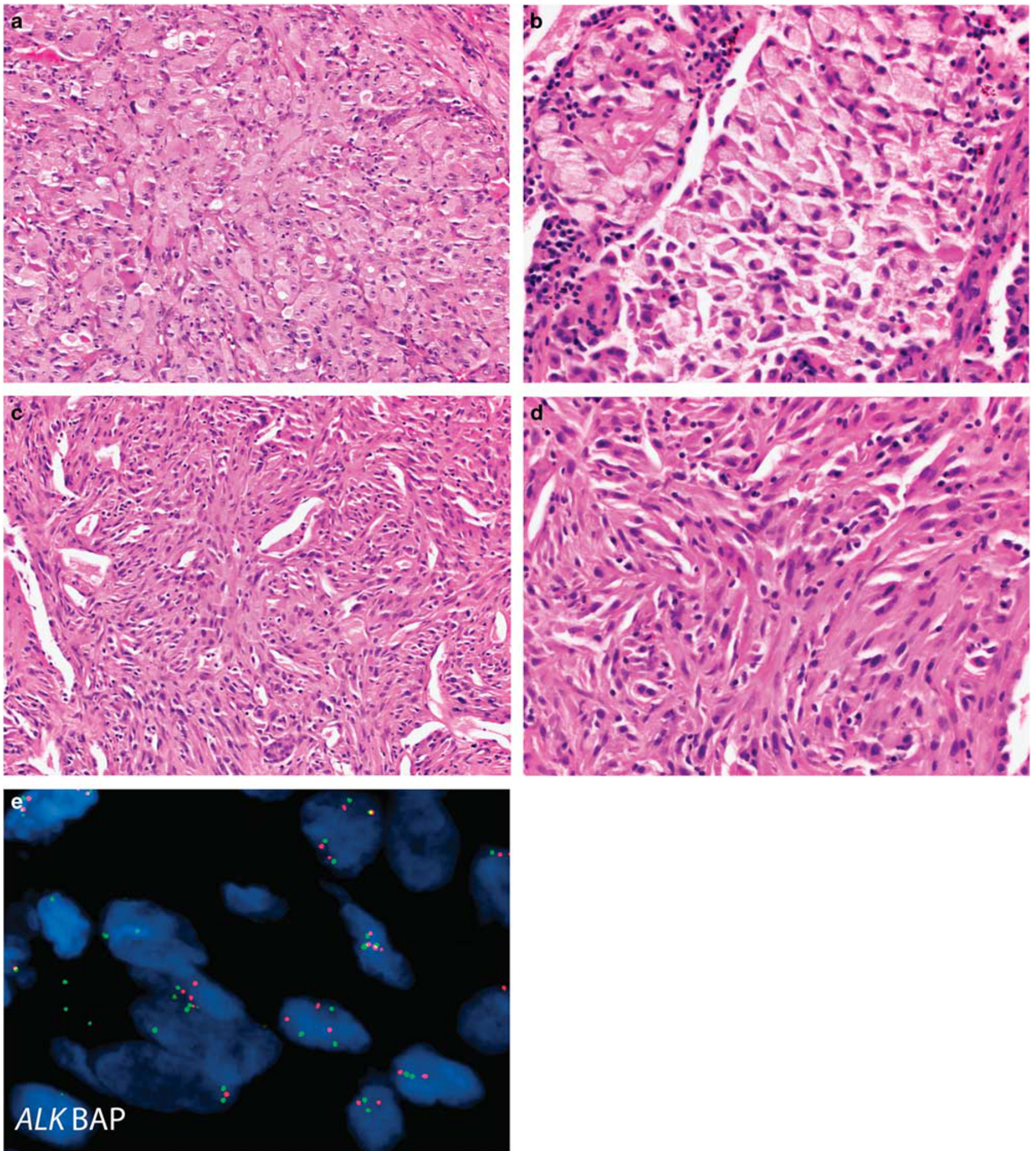

Figure 5 An example of sarcomatoid carcinoma showing $A L K$ mutation alone. The tumor had areas of solid growth (a) as well as foci of signet ring-cell formation (b), which transitioned to areas of high-grade spindle cell morphology (c and d). ALK rearrangement was confirmed by FISH (e, break apart of red and green signals).

molecular analysis of sarcomatoid carcinoma represents an important step toward better understanding of the molecular underpinnings and potential treatment options for this aggressive tumor type.

Our results demonstrate that mutations in the tumor-suppressor gene TP53 are very common in pulmonary sarcomatoid carcinoma, present in $72 \%$ of cases. This is not surprising, given that TP53 mutations are common in various types of highgrade human malignancies, including other types of lung carcinoma, where it has been found in 45-90\% of tumors. ${ }^{27}$ KRAS mutations were frequent as well, present in $30 \%$ of cases, which is very similar to the rate observed in pulmonary adenocarcinomas 
occurring in the US population, which are usually associated with cigarette smoking. ${ }^{12}$ Mutations in KRAS and TP53 have been observed in other studies of pulmonary sarcomatoid carcinoma, ${ }^{22}$ a mutation profile closely resembling that of smoking-related lung adenocaricnoma. The genetic similarity between smoking-related adenocarcinoma and sarcomatoid carcinoma is consistent with histopathological observations that many sarcomatoid carcinomas represent a form of high-grade tumor transition from an underlying adenocarcinoma. Furthermore, KRAS mutations were often observed in conjunction with TP53 mutations in sarcomatoid carcinoma, which are invariably high grade, as opposed to the low-grade mucinous morphology that is classically associated with KRAS mutations alone. ${ }^{28}$ Some of our KRASmutated tumors showed areas of typical mucinous adenocarcinoma, with abrupt transition to high-grade sarcomatoid areas; these relatively 'dedifferentiated' areas may correspond to the accumulation of additional genetic hits associated with tumor progression, including mutations in TP53. However, as the mutations in these morphologically distinct areas of the tumor were not investigated separately, this cannot be confirmed from our data.

Although both TP53 and KRAS mutations are difficult targets for therapeutic intervention, there have been several clinical trials ${ }^{29-31}$ for KRAS mutant adenocarcinomas, as well as preclinical model studies, ${ }^{32}$ with the purpose of optimizing the treatment for this subgroup. Numerous studies have shown various therapeutic approaches for cancers harboring TP53 mutations, ${ }^{33-35}$ including clinical trials specific for lung cancer employing therapies designed to inhibit immune blockade by the tumor (anti-CTLA 4 and anti-PD-1/PD-L1). ${ }^{36}$

Four tumors in our study had mutations in genes with experimental molecular-based therapy for lung carcinoma, namely BRAF, NRAS, PIK3CA, and $A K T 1$. Lung cancer response to the $B R A F$ inhibitors dabrafenib and vemurafenib have been observed in cases reports, ${ }^{37,38}$ and it is also under investigation in clinical trials. ${ }^{12}$ However, the BRAF mutation observed in our study was a non-V600 mutation, which is inactivating and would not be amenable to BRAF inhibition therapy. Limited data have shown that MEK inhibitors may be effective in patients with inactivating BRAF mutations. ${ }^{39}$ NRAS mutations have been found in approximately $1 \%$ of lung carcinoma, ${ }^{40}$ and preclinical models have demonstrated sensitivity to metformin and trametinib combination therapy. ${ }^{41}$ PIK3CA mutations are detected in $2 \%$ of lung carcinomas, ${ }^{42}$ and preclinical data suggest a high sensitivity to PI3K inhibitors. ${ }^{43,44}$ AKT1 mutations have been observed in $1 \%$ of lung adenocarcinomas, ${ }^{12,45}$ and targeting AKT1 using microRNAs have shown in vitro and in vivo suppression of lung tumorigenesis. ${ }^{46}$

One case of $A L K$ rearranged sarcomatoid carcinoma was identified (3\% of total cases), which to our knowledge is quite a rare phenomenon. ${ }^{47,48}$ Thus it seems that rare cases of sarcomatoid carcinoma show mutations in this gene, for which Food and Drug Administration-approved targeted therapies could potentially be used. Presently, $A L K$-rearranged lung adenocarcinomas have two Food and Drug Administration-approved targeted therapies, crizotinib and ceritinib, and a number of nextgeneration ALK tyrosine kinase inhibitors are in clinical development. Crizotinib is a multitargeted tyrosine kinase inhibitor with activity against MET, ALK, and ROS1, that is an effective treatment for many patients with $A L K$-rearranged lung cancer. ${ }^{15,49-52}$ However, virtually all patients develop resistance to crizotinib therapy, usually within 1 year. ${ }^{53-59}$ The higher potency ALK tyrosine kinase inhibitor ceritinib has been studied in both crizotinib-naive and crizotinib-resistant patients and has received Food and Drug Administration approval for crizotinib-resistant or -intolerant $A L K$ rearranged lung carcinoma. ${ }^{17}$ Alectinib is another highly potent and selective ALK inhibitor that has been granted breakthrough-therapy designation by the Food and Drug Administration for ALK-positive advanced lung carcinomas that have progressed on crizotinib therapy. ${ }^{60-63}$

Given that both KRAS and $A L K$ mutations were observed in our sample of sarcomatoid carcinomas, which are genetic abnormalities typically associated with pulmonary adenocarcinoma, it is interesting that no EGFR mutations were observed. EGFR mutations are present in a significant number of pulmonary adenocarcinomas in the United States (15-20\%). This may indicate that adenocarcinomas with EGFR mutations are less likely to undergo high-grade sarcomatoid transformation than tumors with KRAS or $A L K$ mutations, as may be predicted by their often lowgrade nature, regions of lepidic growth, and more indolent course compared with EGFR-negative tumors. It may be that smoking status is also related, as the vast majority of our tumors occurred in smokers, while EGFR-mutated tumors occur in a higher percentage of non-smoking patients. A prior genetic study of sarcomatoid carcinoma performed on Chinese patients did show EGFR mutations present in sarcomatoid carcinoma, ${ }^{64}$ so these mutations can occur in this tumor type, but may be much more uncommon in the United States than in Asian countries where these mutations are known to be more prevalent.

A recent next-generation sequencing study of pulmonary sarcomatoid carcinoma showed MET exon 14 skipping mutations in $22 \%$ of cases. ${ }^{22}$ We did not identify any MET mutations using the cancer hotspot panel in the current study. To further explore this, a manual review of the raw sequencing data was performed specifically looking for MET alterations, but we did not identify any point mutations/indels within the intron/exon boundary at the $5^{\prime}$ end of exon 14 . The $3^{\prime}$ end (including exon/ intron boundary) of exon 14 was not interrogated with our hotspot panel. The discordant results 
between the current study and the previously published study may be explained by the different next-generation sequencing methodologies (ie, deep sequencing of targeted regions vs wide-scale genomic sequencing) and is a potential limitation of our study method.

In summary, our next-generation sequencing-based analysis of common mutations revealed that pulmonary sarcomatoid carcinomas have a genetic phenotype very similar to that of high-grade lung adenocarcinoma from smokers, characterized by frequent TP53 and KRAS gene mutations present in the same tumor. We identified one sarcomatoid tumor $(3 \%)$ with an $A L K$ translocation, which could have benefited from targeted therapy. Although sarcomatoid carcinomas are a rare pulmonary neoplasm, our results suggest that they should be evaluated for potential targetable mutations in the same manner as adenocarcinomas, to improve therapeutic options for this aggressive form of lung cancer.

\section{Disclosure/conflict of interest}

The authors declare no conflict of interest.

\section{References}

1 Fishback NF, Travis WD, Moran CA et al. Pleomorphic (spindle/giant cell) carcinoma of the lung. A clinicopathologic correlation of 78 cases. Cancer 1994;73: 2936-2945.

2 Nakajima M, Kasai T, Hashimoto $\mathrm{H}$ et al. Sarcomatoid carcinoma of the lung: a clinicopathologic study of 37 cases. Cancer 1999;86:608-616.

3 Rossi G, Cavazza A, Sturm N et al. Pulmonary carcinomas with pleomorphic, sarcomatoid, or sarcomatous elements: a clinicopathologic and immunohistochemical study of 75 cases. Am J Surg Pathol 2003;27: 311-324.

4 Martin LW, Correa AM, Ordonez NG et al. Sarcomatoid carcinoma of the lung: a predictor of poor prognosis. Ann Thorac Surg 2007;84:973-980.

5 Huang SY, Shen SJ, Li XY. Pulmonary sarcomatoid carcinoma: a clinicopathologic study and prognostic analysis of 51 cases. World J Surg Oncol 2013;11:252.

6 Lin Y, Yang H, Cai Q et al. Characteristics and prognostic analysis of 69 patients with pulmonary sarcomatoid carcinoma. Am J Clin Oncol 2014 (e-pub ahead of print).

7 Ouziane I, Boutayeb S, Mrabti H et al. Sarcomatoid carcinoma of the lung: a model of resistance of chemotherapy. North Am J Med Sci 2014;6:342-345.

8 Kerr K, Pelosi G, Austin JHM et al. Pleomorphic, spindle cell, and giant cell carcinoma; cacinosarcoma; and pulmonary blastoma. In: Travis WD, Brambilla E, Burke AP et al (eds). WHO Classification of Tumours of the Lung, Pleura, Thymus and Heart. IARC Press: Lyon, France, 2015, pp 88-94.

9 Nappi O, Glasner SD, Swanson PE et al. Biphasic and monophasic sarcomatoid carcinomas of the lung. A reappraisal of 'carcinosarcomas' and 'spindle-cell carcinomas'. Am J Clin Pathol 1994;102:331-340.
10 Chang YL, Lee YC, Shih JY et al. Pulmonary pleomorphic (spindle) cell carcinoma: peculiar clinicopathologic manifestations different from ordinary nonsmall cell carcinoma. Lung Cancer 2001;34:91-97.

11 Li T, Kung HJ, Mack PC et al. Genotyping and genomic profiling of non-small-cell lung cancer: implications for current and future therapies. J Clin Oncol 2013;31: 1039-1049.

12 Moreira AL, Eng J. Personalized therapy for lung cancer. Chest 2014;146:1649-1657.

13 Shames DS, Wistuba II. The evolving genomic classification of lung cancer. J Pathol 2014;232:121-133.

14 Devarakonda S, Morgensztern D, Govindan R. Genomic alterations in lung adenocarcinoma. Lancet Oncol 2015;16:e342-e351.

15 Malik SM, Maher VE, Bijwaard KE et al. U.S. Food and Drug Administration approval: crizotinib for treatment of advanced or metastatic non-small cell lung cancer that is anaplastic lymphoma kinase positive. Clin Cancer Res 2014;20:2029-2034.

16 Shaw AT, Solomon B, Kenudson MM. Crizotinib and testing for ALK. J Natl Compr Canc Netw 2011;9: 1335-1341.

17 Friboulet L, Li N, Katayama R et al. The ALK inhibitor ceritinib overcomes crizotinib resistance in non-small cell lung cancer. Cancer Discov 2014;4:662-673.

18 Pao W, Miller V, Zakowski M et al. EGF receptor gene mutations are common in lung cancers from 'never smokers' and are associated with sensitivity of tumors to gefitinib and erlotinib. PNAS 2004;101:13306-13311.

19 Lynch TJ, Bell DW, Sordella R et al. Activating mutations in the epidermal growth factor receptor underlying responsiveness of non-small-cell lung cancer to gefitinib. N Engl J Med 2004;350:2129-2139.

20 Paez JG, Janne PA, Lee JC et al. EGFR mutations in lung cancer: correlation with clinical response to gefitinib therapy. Science 2004;304:1497-1500.

21 Dungo RT, Keating GM. Afatinib: first global approval. Drugs 2013;73:1503-1515.

22 Liu X, Jia Y, Stoopler MB et al. Next-generation sequencing of pulmonary sarcomatoid carcinoma reveals high frequency of actionable MET gene mutations. J Clin Oncol 2016;34:794-802.

23 Frampton GM, Ali SM, Rosenzweig M et al. Activation of MET via diverse exon 14 splicing alterations occurs in multiple tumor types and confers clinical sensitivity to MET inhibitors. Cancer Discov 2015;5:850-859.

24 Terra SB, Aubry MC, Yi ES et al. Immunohistochemical study of 36 cases of pulmonary sarcomatoid carcinoma-sensitivity of TTF-1 is superior to napsin. Hum Pathol 2014;45:294-302.

25 Jang JS, Lee A, Li J et al. Common oncogene mutations and novel SND1-BRAF transcript fusion in lung adenocarcinoma from never smokers. Sci Rep 2015;5:9755.

26 Lozano R, Naghavi M, Foreman K et al. Global and regional mortality from 235 causes of death for 20 age groups in 1990 and 2010: a systematic analysis for the Global Burden of Disease Study 2010. Lancet 2012;380: 2095-2128.

27 Chiba I, Takahashi T, Nau MM et al. Mutations in the p53 gene are frequent in primary, resected non-small cell lung cancer. Oncogene 1990;5:1603-1610.

28 Kakegawa S, Shimizu K, Sugano M et al. Clinicopathological features of lung adenocarcinoma with KRAS mutations. Cancer 2011;117:4257-4266.

29 Adjei AA, Mauer A, Bruzek L et al. Phase II study of the farnesyl transferase inhibitor R115777 in patients with 
advanced non-small-cell lung cancer. J Clin Oncol 2003;21:1760-1766.

30 Janne PA, Shaw AT, Pereira JR et al. Selumetinib plus docetaxel for KRAS-mutant advanced non-small-cell lung cancer: a randomised, multicentre, placebo-controlled, phase 2 study. Lancet Oncol 2013;14:38-47.

31 Konstantinidou G, Bey EA, Rabellino A et al. Dual phosphoinositide 3-kinase/mammalian target of rapamycin blockade is an effective radiosensitizing strategy for the treatment of non-small cell lung cancer harboring K-RAS mutations. Cancer Res 2009;69:7644-7652.

32 Ostrem JM, Peters U, Sos ML et al. K-Ras(G12C) inhibitors allosterically control GTP affinity and effector interactions. Nature 2013;503:548-551.

33 Roth JA, Nguyen D, Lawrence DD et al. Retrovirusmediated wild-type p53 gene transfer to tumors of patients with lung cancer. Nat Med 1996;2:985-991.

34 Bykov VJ, Issaeva N, Shilov A et al. Restoration of the tumor suppressor function to mutant p53 by a lowmolecular-weight compound. Nat Med 2002;8:282-288.

35 Bykov VJ, Zache N, Stridh H et al. PRIMA-1(MET) synergizes with cisplatin to induce tumor cell apoptosis. Oncogene 2005;24:3484-3491.

36 Pardoll DM. The blockade of immune checkpoints in cancer immunotherapy. Nat Rev Cancer 2012;12: $252-264$.

37 Schmid S, Siano M, Joerger $M$ et al. Response to dabrafenib after progression on vemurafenib in a patient with advanced BRAF V600E-mutant bronchial adenocarcinoma. Lung Cancer 2015;87:85-87.

38 Robinson SD, O'Shaughnessy JA, Cowey CL et al. BRAF V600E-mutated lung adenocarcinoma with metastases to the brain responding to treatment with vemurafenib. Lung Cancer 2014;85:326-330.

39 Heidorn SJ, Milagre C, Whittaker S et al. Kinase-dead BRAF and oncogenic RAS cooperate to drive tumor progression through CRAF. Cell 2010;140:209-221.

40 Ohashi K, Sequist LV, Arcila ME et al. Characteristics of lung cancers harboring NRAS mutations. Clin Cancer Res 2013;19:2584-2591.

41 Vujic I, Sanlorenzo M, Posch C et al. Metformin and trametinib have synergistic effects on cell viability and tumor growth in NRAS mutant cancer. Oncotarget 2015;6:969-978.

42 Kawano O, Sasaki H, Endo K et al. PIK3CA mutation status in Japanese lung cancer patients. Lung Cancer 2006;54:209-215.

43 Engelman JA, Chen L, Tan X et al. Effective use of PI3K and MEK inhibitors to treat mutant Kras G12D and PIK3CA H1047R murine lung cancers. Nature Med 2008;14:1351-1356.

44 Lee H, Kim SJ, Jung KH et al. A novel imidazopyridine PI3K inhibitor with anticancer activity in non-small cell lung cancer cells. Oncol Reports 2013;30:863-869.

45 De Marco C, Malanga D, Rinaldo N et al. Mutant AKT1$\mathrm{E} 17 \mathrm{~K}$ is oncogenic in lung epithelial cells. Oncotarget 2015;6:39634-39650.

46 Yoo JK, Jung HY, Lee JM et al. The novel miR-9500 regulates the proliferation and migration of human lung cancer cells by targeting AKT1. Cell Death Differ 2014;21:1150-1159.

47 Ali G, Proietti A, Niccoli C et al. EML4-ALK translocation in both metachronous second primary lung sarcomatoid carcinoma and lung adenocarcinoma: a case report. Lung Cancer 2013;81:297-301.
48 Kobayashi Y, Sakao Y, Ito $\mathrm{S}$ et al. Transformation to sarcomatoid carcinoma in ALK-rearranged adenocarcinoma, which developed acquired resistance to crizotinib and received subsequent chemotherapies. J Thorac Oncol 2013;8:e75-e78.

49 Kwak EL, Bang YJ, Camidge DR et al. Anaplastic lymphoma kinase inhibition in non-small-cell lung cancer. N Engl J Med 2010;363:1693-1703.

50 Camidge DR, Bang YJ, Kwak EL et al. Activity and safety of crizotinib in patients with ALK-positive nonsmall-cell lung cancer: updated results from a phase 1 study. Lancet Oncol 2012;13:1011-1019.

51 Shaw AT, Kim DW, Nakagawa K et al. Crizotinib versus chemotherapy in advanced ALK-positive lung cancer. N Engl J Med 2013;368:2385-2394.

52 Solomon BJ, Mok T, Kim DW et al. First-line crizotinib versus chemotherapy in ALK-positive lung cancer. N Engl J Med 2014;371:2167-2177.

53 Choi YL, Soda M, Yamashita Y et al. EML4-ALK mutations in lung cancer that confer resistance to ALK inhibitors. N Engl J Med 2010;363:1734-1739.

54 Heuckmann JM, Holzel M, Sos ML et al. ALK mutations conferring differential resistance to structurally diverse ALK inhibitors. Clin Cancer Res 2011;17: 7394-7401.

55 Sasaki T, Koivunen J, Ogino A et al. A novel ALK secondary mutation and EGFR signaling cause resistance to ALK kinase inhibitors. Cancer Res 2011;71: 6051-6060.

56 Costa DB, Kobayashi S, Pandya SS et al. CSF concentration of the anaplastic lymphoma kinase inhibitor crizotinib. J Clin Oncol 2011;29:e443-e445.

57 Doebele RC, Pilling AB, Aisner DL et al. Mechanisms of resistance to crizotinib in patients with ALK gene rearranged non-small cell lung cancer. Clin Cancer Res 2012;18:1472-1482.

58 Katayama R, Shaw AT, Khan TM et al. Mechanisms of acquired crizotinib resistance in ALK-rearranged lung Cancers. Science Transl Med 2012:4:120ra17.

59 Steuer CE, Ramalingam SS. ALK-positive non-small cell lung cancer: mechanisms of resistance and emerging treatment options. Cancer 2014;120:2392-2402.

60 Sakamoto H, Tsukaguchi T, Hiroshima S et al. CH5424802, a selective ALK inhibitor capable of blocking the resistant gatekeeper mutant. Cancer Cell 2011;19:679-690.

61 Seto T, Kiura K, Nishio M et al. CH5424802 (RO5424802) for patients with ALK-rearranged advanced non-small-cell lung cancer (AF-001JP study): a single-arm, open-label, phase 1-2 study. Lancet Oncol 2013;14:590-598.

62 Kodama T, Tsukaguchi T, Yoshida M et al. Selective ALK inhibitor alectinib with potent antitumor activity in models of crizotinib resistance. Cancer Lett 2014;351:215-221.

63 Gadgeel SM, Gandhi L, Riely GJ et al. Safety and activity of alectinib against systemic disease and brain metastases in patients with crizotinib-resistant ALKrearranged non-small-cell lung cancer (AF-002JG): results from the dose-finding portion of a phase 1/2 study. Lancet Oncol 2014;15:1119-1128.

64 Jiang X, Liu Y, Chen C et al. The value of biomarkers in patients with sarcomatoid carcinoma of the lung: molecular analysis of 33 cases. Clin Lung Cancer 2012;13:288-296. 